\title{
Iron deficiency-induced thrombocytosis increases thrombotic tendency in rats
}

Haematologica 2021

Volume 106(3):782-794

\section{Correspondence:}

CHRISTOPH GASCHE

christoph.gasche@meduniwien.ac.at

Received: December 13, 2019.

Accepted: February 17, 2020.

Pre-published: February 20, 2020.

https://doi.org/10.3324/haematol.2019.245092

(C)2021 Ferrata Storti Foundation

Material published in Haematologica is covered by copyright. All rights are reserved to the Ferrata Storti Foundation. Use of published material is allowed under the following terms and conditions:

https://creativecommons.org/licenses/by-nc/4.0/legalcode. Copies of published material are allowed for personal or internal use. Sharing published material for non-commercial purposes is subject to the following conditions:

https://creativecommons.org/licenses/by-nc/4.0/leǵalcode, sect. 3. Reproducing and sharing published material for commercial purposes is not allowed without permission in writing from the publisher.

\begin{abstract}
Kristine Jimenez, ${ }^{1}$ Florentina Leitner, ${ }^{1}$ Aran Leitner, ${ }^{1}$ Gisela Scharbert, ${ }^{2}$ Philipp Schwabl, ${ }^{1}$ Anne-Margarethe Kramer, ${ }^{3}$ Anita Krnjic, ${ }^{1}$ Joachim Friske, ${ }^{4}$ Thomas Helbich, ${ }^{4}$ Rayko Evstatiev, ${ }^{1}$ Vineeta Khare $^{1}$ and Christoph Gasche ${ }^{1}$

${ }^{1}$ Division of Gastroenterology \& Hepatology, Department of Internal Medicine III, Medical University of Vienna; '2Department of Special Anesthesiology and Pain Management, General Intensive Care and Pain Control, Medical University of Vienna; ${ }^{3}$ Department of Biomedical Research, Medical University of Vienna and ${ }^{4}$ Department of Biomedical Imaging and Image-guided Therapy, Division of Gender and Molecular Imaging, Medical University of Vienna, Vienna, Austria
\end{abstract}

\section{ABSTRACT}

Trors ron deficiency (ID) is globally prevalent, and apart from anemia is associated with thrombocytosis. While considered benign, studies linking thrombotic events with prior ID anemia suggest otherwise. In this study we used animal models to assess the influence of ID on thrombotic tendency. Sprague-Dawley rats were fed control or iron-deficient diets and ferric carboxymaltose was used to reverse ID. Thrombosis was induced by stenosis of the inferior vena cava or damage to the right carotid artery using ferric chloride. Thrombi were evaluated histologically and by high frequency ultrasound in the venous model. ID consistently induced thrombocytosis alongside anemia. The growth of venous thrombi and the final dimensions of both arterial and venous thrombi were greater in animals with ID. In both models, platelet numbers correlated with the final thrombus size, with thrombi in iron-deficient animals having the largest platelet areas. Platelet function was also evaluated in surgically-naïve rats. Coagulability, determined by thromboelastography, and hemostasis, evaluated by tail transection, were enhanced in the animals with ID. Platelet P-selectin expression and plasma P-selectin levels were both higher in animals with ID. Platelet adhesion and aggregation in ID was impaired under shear flow but was intact in static assays. Iron replacement therapy reversed all ID-related changes in hematologic parameters, thrombus dimensions, and platelet assays. In summary, ID alone increases thrombotic tendency. Iron replacement therapy reverses these changes, making it a viable strategy for the prevention of ID-related thrombotic disease. This may be of importance in patients with chronic illnesses who may already be at increased risk of thrombosis, such as those with inflammatory bowel disease, chronic kidney disease, or cancer.

\section{Introduction}

Iron-deficiency anemia (IDA) affects over 1.2 billion people worldwide, with perhaps just as many having iron deficiency (ID) without manifest anemia. ${ }^{1}$ Iron is essential, being vital to oxygen transport via hemoglobin, mitochondrial function, DNA replication and repair, cellular metabolism and signaling, and it even plays a role in host defenses. ${ }^{2}$ Thus, apart from anemia, ID has been connected to impaired cognition and muscular function, and is detrimental in the context of cardiovascular disease. ${ }^{1,3} \mathrm{ID}$ is also a known cause of thrombocytosis, although the pathological consequences of this phenomenon have not been well studied. ${ }^{4}$

Numerous studies have linked thrombotic events with prior IDA..$^{5 \cdot 11}$ In adults, patients with ischemic stroke or venous thromboembolism (VTE) were about 1.4 times more likely to have had prior IDA. ${ }^{10,11}$ In children, in whom ID is more common, prior IDA was 3.8 to 10 times more likely in those who suffered a stroke., Platelet counts were also higher in patients with stroke and IDA. In one prospective 
study in patients with unprovoked VTE, recurrence of VTE was more likely in the presence of ID. ${ }^{12}$ A recent large, retrospective study found that patients with IDAassociated thrombocytosis had a 2-fold higher risk of thrombosis in comparison to those with a normal platelet count. ${ }^{13}$ In cancer, a high platelet count is an independent risk factor for VTE. ${ }^{14}$ Erythropoiesis-stimulating agents increase thrombotic risk in cancer, but the simultaneous administration of intravenous iron lowers the incidence of VTE and the platelet count, ostensibly through prevention of iron-restricted erythropoiesis. ${ }^{15}$

Collectively, these studies suggest that ID-associated thrombocytosis may not be entirely benign. Given the widespread prevalence of ID, the impact of this complication could be significant. Furthermore, ID may be co-morbid to conditions which already predispose towards thrombosis, such as inflammatory bowel disease, chronic kidney disease, chronic heart failure, or cancer. Chronic illness leads to the induction of hepcidin, which causes the sequestration of iron from the circulation. ${ }^{1,2}$ The combination of the disease and ID may thus further increase thrombotic risk.

We previously showed, in an animal model, that ID leads to thrombocytosis by augmenting megakaryopoiesis. ${ }^{16,17}$ Our group has also shown that iron replacement therapy reverses ID-associated thrombocytosis in inflammatory bowel disease, without altering inflammatory parameters. ${ }^{18,19}$ In the current study, we investigated whether ID alone could increase thrombotic tendency. We found that ID consistently induced thrombocytosis, augmented some aspects of platelet function, and ultimately increased thrombus size in both venous and arterial models of thrombosis. Iron administration reversed these changes, underscoring the direct relationship with ID and emphasizing the importance of maintaining iron homeostasis.

\section{Methods}

Ethical approval was obtained from the Austrian Federal Ministry for Science, Research, and Economy and experiments followed guidelines for Good Scientific Practice of the Medical University of Vienna.

Male 4- to 5-week old Sprague-Dawley rats (Himberg, Austria and Charles River Laboratories, Germany) were fed either a control diet (Con: E15510-04, $196 \mathrm{mg} / \mathrm{kg} \mathrm{Fe}$, Ssniff) or iron-deficient diet (Def: $<10 \mathrm{mg} / \mathrm{kg}$ Fe, E155102404, Ssniff). Ferric carboxymaltose at a dose of 5, 10, or $20 \mathrm{mg} / \mathrm{kg}$ body weight (BW) $(+\mathrm{Fe} 5,+\mathrm{Fe} 10,+\mathrm{Fe} 20$, respectively) or placebo $(0.9 \% \mathrm{NaCl})$ was administered intraperitoneally to Def animals in the third, fourth and fifth weeks to determine the optimal dosage. Con animals received placebo. Subsequent experiments used $20 \mathrm{mg} / \mathrm{kg}$ BW ferric carboxymaltose (Def $+\mathrm{Fe}$ ).

Prior to and during surgery, animals were anesthetized (see Online Supplementary Material). In order to induce venous thrombosis, inferior vena cava (IVC) branches between the left renal and caudal and iliac veins were ligated or cauterized to avoid collateral flow disruption of thrombus formation. ${ }^{20}$ The IVC and an interposing 2-prolene suture were ligated below the left renal vein using a 7-0 silk suture. The 2 -prolene suture was removed, allowing residual flow. High frequency ultrasound was performed using a Vevo 2100 imaging station (Fujifilm
Visualsonics, Amsterdam, the Netherlands), with an MS250 transducer (13-24 MHz) and three-dimensional motor stage. Thrombi were circumscribed at multiple two-dimensional cross-sectional views for three-dimensional reconstruction. Four hours after ligation, IVC containing thrombi were collected for histology.

To induce arterial thrombosis, a filter paper soaked in $10 \% \mathrm{FeCl}_{3}$ was placed on the right common carotid artery for $5 \mathrm{~min}$, and then removed. Flow was monitored using a distally placed flow probe (Transonic, Ithaca, NY, USA) until occlusion. ${ }^{21}$ Thereafter, arteries with thrombi were excised for histology.

For histology, Carstairs staining was performed as previously described. ${ }^{22}$ Immunohistochemistry was performed for ITGA2B (1:200, Abcam, Cambridge, UK), and von Willebrand factor (1:1000, Sigma-Aldrich). Thrombi were imaged with an Olympus BX61US Slide Scanner and analyzed using Fiji/ImageJ..$^{23,24}$ Liver and spleen were stained with the standard Perls Prussian blue stain and imaged using an Olympus BX41 microscope.

\section{Platelet function tests}

Blood was collected via cardiac puncture from surgically naïve animals. Platelet function was assessed by aggregation to ADP or collagen (Multiplate aggregometer, Cobas, Roche, Switzerland), adhesion under flow using the Collagen/ADP kit (Platelet Function Analyzer 100 [PFA100], Siemens, Germany), and thromboelastography (TEG 5000, Haemonetics, MA, USA). Washed platelets were used to test static adhesion to wells coated with $1 \mathrm{mg} / \mathrm{mL}$ fibrinogen (Sigma-Aldrich) or $1 \mathrm{mg} / \mathrm{mL}$ collagen (Thermofisher, MA, USA). ${ }^{1}$

Flow cytometry was conducted on sublingual anticoagulated blood. ${ }^{25}$ Platelets were labeled with CD61-FITC (ebioscience, Thermofisher) and their activity measured using CD62P-PE (P-selectin, ebioscience, Thermofisher). The expression of CD62P was evaluated at baseline and after stimulation with thrombin $(0.016 \mathrm{U} / \mathrm{mL}, 0.25 \mathrm{U} / \mathrm{mL}$, Sigma) on a Cell Lab Quanta SC (Beckman Coulter, CA, USA).

\section{Statistical analysis}

A t-test for independent samples or analysis of variance with Tukey post-hoc testing was used to analyze variables with a normal distribution. Otherwise, Kruskall-Wallis and Mann-Whitney U tests with Bonferonni-Holm correction for multiple comparisons were used. Correlations were tested using the Pearson correlation or Spearman rho as appropriate. Statistical testing was performed using SPSS 23.

\section{Results}

\section{Iron deficiency causes thrombocytosis, which is reversed by iron replacement therapy in a dose-dependent fashion}

Our group previously established a model of dietinduced ID that leads to anemia and thrombocytosis. ${ }^{16}$ Here, we evaluated the reversibility of these changes upon administration of a commonly used parenteral iron, ferric carboxymaltose.

Animals were placed on an iron-deficient diet (Def) for 3 weeks, and then given weekly injections of ferric carboxymaltose at a dose of 5,10 , or $20 \mathrm{mg} / \mathrm{kg}$ BW or place- 
A

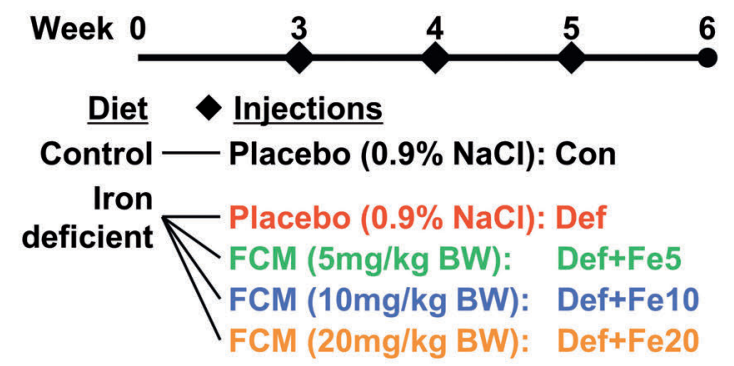

C
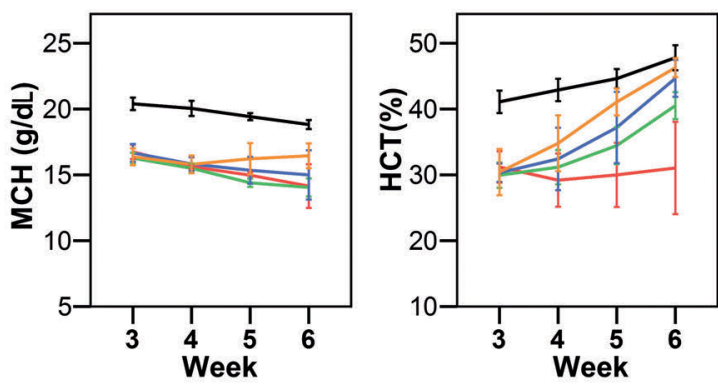
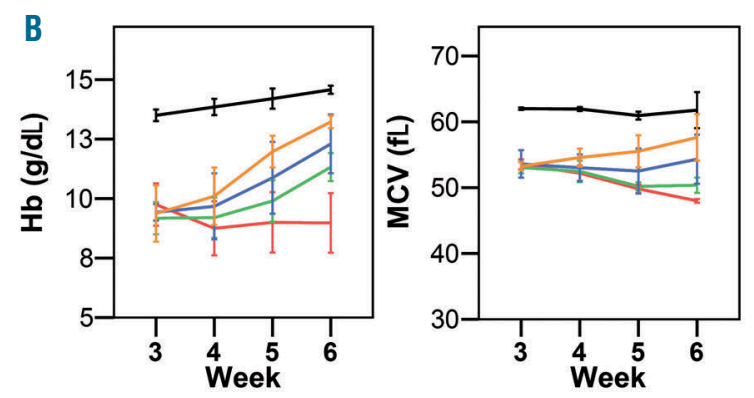

D
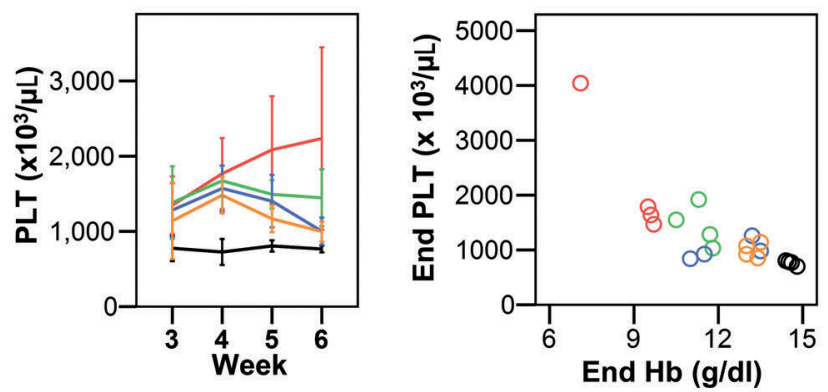

E

Prussian Blue Staining
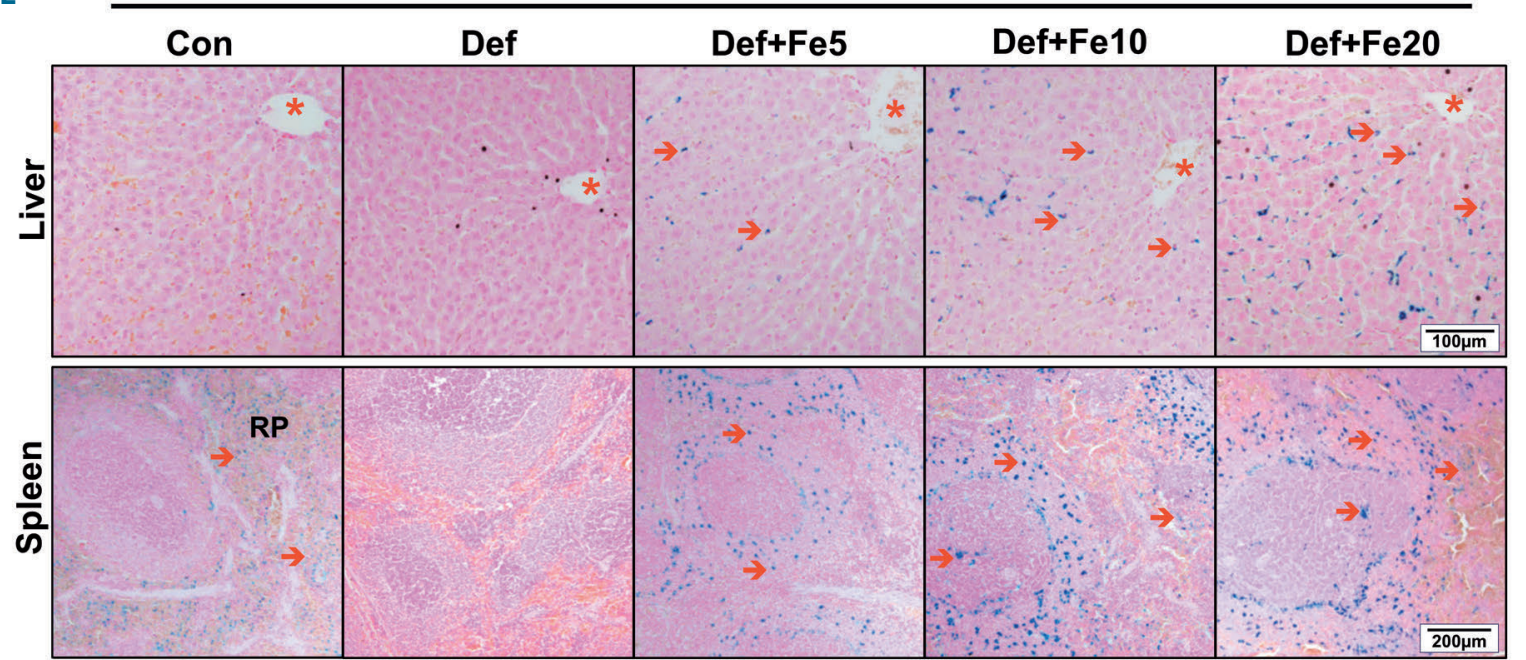

$\mathrm{F}$

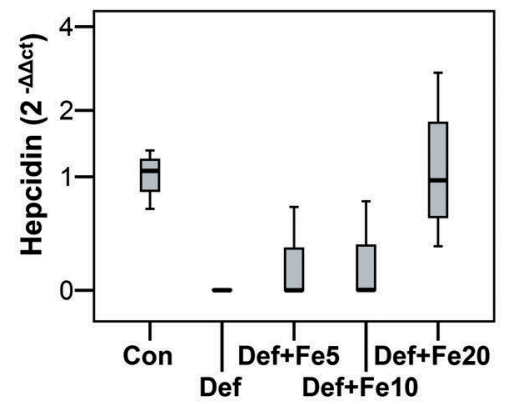

G

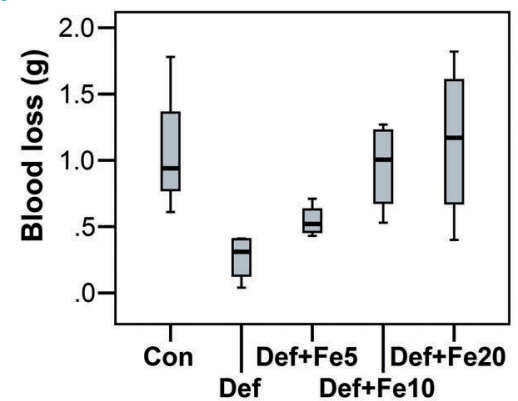

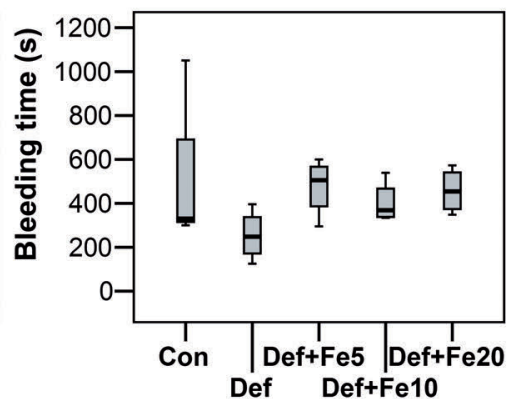

Figure 1. Iron deficiency causes thrombocytosis, which is reversed by iron replacement therapy in a dose-dependent fashion. (A) Experimental design. Rats were fed an iron-deficient diet (Def) for 6 weeks, and given three injections of ferric carboxymaltose at the doses of 5,10 , or $20 \mathrm{mg} / \mathrm{kg}$ body weight (BW, +Fe5, +Fe10, $+\mathrm{Fe} 20$, respectively) or placebo $(4 \mathrm{~mL} / \mathrm{kg}$ BW $0.9 \% \mathrm{NaCl}$ ). Animals fed a control diet and given injections of placebo formed the control group (Con). Hematologic parameters were measured weekly from 3 to 6 weeks after the start of the experiment ( $n=4$ per group). (B). Changes in hemoglobin and mean corpuscular volume from 3 to 6 weeks in the different groups of animals. (C) Values of mean corpuscular hemoglobin, hematocrit and platelet count over the course of the experiment. (D) Platelet counts versus hemoglobin concentration at 6 weeks. (E) Representative images of liver and spleen histological staining for iron (Prussian blue stain). Red arrows mark positively stained cells. Red asterisks $\left(^{*}\right)$ mark central veins. (F) Relative expression of hepcidin as determined by real-time quantitative polymerase chain reaction analysis of liver isolates. Values are normalized to those in Con animals, with Hprt1 as the endogenous control. ( $n=3-4$ per group). (G) Blood loss and bleeding time measured during the tail bleeding assay ( $n=3-4$ per group). Error bars: mean \pm standard deviation. FCM: ferric carboxymaltose, Hb: hemoglobin, MCV: mean corpuscular volume, $\mathrm{MCH}$ : mean corpuscular hemoglobin, HCT: hematocrit, PLT: platelet count 
bo $(0.9 \% \mathrm{NaCl})$ at weeks 3,4 and 5 . Control animals (Con) were given a normal diet and placebo injections (Figure 1A). At 3 weeks, Def animals were anemic (hemoglobin: $9.4 \pm 0.8 \mathrm{~g} / \mathrm{dL}$ ) in comparison to Con animals (hemoglobin: $13.5 \pm 0.2 \mathrm{~g} / \mathrm{dL}$ ). Both mean corpuscular volume (Def: 53.1 [IOR: 52.7-54] fl vs. Con: 62 [IOR: 61.8$62.2] \mathrm{fL}$ ) and mean corpuscular hemoglobin (Def: $16.5 \pm 0.6$ g/dL vs. Con: $20.4 \pm 0.5 \mathrm{~g} / \mathrm{dL}$ ) were likewise lower, consistent with the microcytic and hypochromic anemia of ID. As in previous studies, ${ }^{16}$ platelet counts were higher in Def rats $\left(1288.8 \pm 403.2 \times 10^{3} / \mu \mathrm{L}\right)$ than in Con animals $\left(776.3 \pm 171.6 \times 10^{3} / \mu \mathrm{L}\right)$ (Figure $1 \mathrm{~B}$ and $\mathrm{C}$, Online Supplementary Table S1).

Over the following weeks, there were dose- and timedependent improvements in the affected erythroid and platelet hematologic parameters, with no changes in white blood cell counts (Figure $1 \mathrm{~B}$ and C, Online Supplementary Figure S1A, Online Supplementary Table S1). Weight and food consumption were comparable between groups (Online Supplementary Figure $S 1 B$ ). We found a strong correlation between hemoglobin concentration and platelet count at the end of the experiment $(r=0.812$, $P<0.001$ ) (Figure 1D). A dose of $20 \mathrm{mg} / \mathrm{kg}$ BW ferric carboxymaltose was the most effective at normalizing hematologic parameters, as those of Def $+\mathrm{Fe} 20$ animals were closest to those of Con rats at the end of the experiment. Def animals remained anemic, with elevated platelet counts (Figure 1B and C, Online Supplementary Table S1).

After Prussian blue staining, iron and could be found in cells lining liver sinusoids, with staining extending further towards central veins (Figure 1E) with increasing dose. In spleens, stainable iron was found in the red pulp of Con animals but was completely absent in Def rats. Accumulation in the marginal zone increased with higher iron dosage. Among Def animals, positive staining for iron in the red pulp, the site of erythropoiesis, was most prominent in $\mathrm{Def}+\mathrm{Fe} 20$ rats.

The levels of hepcidin, the central regulator of systemic iron homeostasis, are low during ID. The expression of liver hepcidin, as determined by real-time quantitative polymerase chain reaction, was low in all Def animals, except for Def+Fe20 ones, in which it approached the values in Con animals (Figure 1F). Based on these results, the dose of ferric carboxymaltose selected for subsequent experiments was $20 \mathrm{mg} / \mathrm{kg} \mathrm{BW}$, as this was the dose most effective at reversing ID.

Our animal model clearly showed that ID causes thrombocytosis; however it was not clear whether this has any mechanistic consequences. We first evaluated hemostasis using the tail bleeding assay. Def rats had a shorter bleeding time and less blood loss as compared to Con rats (Figure 1G). Iron replacement therapy increased blood loss in a dose-dependent manner, with the loss approaching levels of Con animals at higher concentrations of iron. This supports the notion that ID-induced thrombocytosis is an adaptive mechanism to counter potential blood loss. On the other hand, ID-induced thrombocytosis may contribute to an increased tendency to thrombosis.

\section{Iron deficiency enhances thrombotic tendency in a venous model of thrombosis}

To evaluate a thrombotic tendency in ID, we utilized a model of venous thrombosis induced by stenosis of the IVC. We initially evaluated the effect of ID alone (Experiment 1, Figure 2), and then reversed ID with the established dose of ferric carboxymaltose (Experiment 2, Figure 3). As expected, in experiment 1 Def animals developed microcytic hypochromic anemia alongside thrombocytosis (Figure 2A-D, Online Supplementary Table S2).

After induction of thrombosis, high frequency ultrasonography was performed along the full length of the thrombus in vivo (Online Supplementary Figure S2A, Online Supplementary Video 1). The thrombus circumference was outlined on multiple cross-sectional planes (Online Supplementary Figure S2B), enabling three-dimensional reconstruction and calculation of thrombus dimensions (Online Supplementary Figure S2D and E)

In preliminary experiments with untreated animals, we observed that thrombus volume did not increase in size beyond $24 \mathrm{~h}$ after ligation and shrank, likely reflecting thrombus resolution (Online Supplementary Figure S3A). As we were interested in thrombus formation, we evaluated thrombus size in the hours shortly after IVC stenosis and found that thrombus size values formed a plateau from 5 h (Online Supplementary Figure S3B). Hypothesizing that thrombus formation would be accelerated in ID, we selected $4 \mathrm{~h}$ after stenosis as our endpoint for comparing iron-treated animals to Con animals.

Upon induction of venous thrombosis, resultant thrombi were larger in Def rats than in Con rats at $3 \mathrm{~h}$ and $4 \mathrm{~h}$, as determined by both ultrasound, and histology (Figure 2E-K, Online Supplementary Figure S4A). The increase in thrombus volume from 3 to $4 \mathrm{~h}$ was significantly larger in Def rats $\left(73.4 \pm 38.7 \mathrm{~mm}^{3}\right.$ vs. Con: $\left.21 \pm 9.2 \mathrm{~mm}^{3}, P=0.019\right)$ (Figure 2G) and the final volume at $4 \mathrm{~h}$ was likewise larger in these animals (Def: $208.2 \pm 74.9 \mathrm{~mm}^{3}$ vs. Con: $81.6 \pm$ $20.5 \mathrm{~mm}^{3}, P=0.008$ ) (Figure $2 \mathrm{H}$ ). Thrombus length followed a similar pattern although the increase in length from 3 to $4 \mathrm{~h}$ was not statistically significant (Def: 9.87 [IQR: 9.09-12.3] mm vs. Con: -0.35 [IQR: -0.85-3.3] mm, $P=0.117$ ) (Figure 2I and J), because of one Def animal whose thrombus did not increase in length within the period of measurement. The final thrombus was, however, significantly longer in Def rats $(30.4 \pm 82 \mathrm{~mm}$, vs. Con: $15 \pm 3.4 \mathrm{~mm}, P=0.004)$. Thrombus area was also measured by $B$-mode transverse ultrasound and found to be larger in Def animals (62.9 [IOR:56.5-74.7] $\mathrm{mm}^{2}$ vs. Con: 39.4 [IOR: 33.6-54.6] $\mathrm{mm}^{2} ; P=0.007$ ) (Online Supplementary Figure $S 4 B$, $C)$. On histology, the transverse area of the thrombi was larger in Def rats (41.5 [IOR: 39.9-55.8] $\mathrm{mm}^{2}$ vs. Con: 25.3 [IOR: 9.1-21.5] $\mathrm{mm}^{2}, P=0.001$ ) (Figure 2L). There was a strong correlation between histologically determined area and ultrasound-measured volume $(r=0.912, P \leq 0.001)$ (Figure $2 \mathrm{M}$ ). The length of the thrombi determined histologically was also larger in Def animals $(19.2 \pm 4.3 \mathrm{~mm}$ vs. Con: $13 \pm 3.5 \mathrm{~mm}, P=0.005)$, and likewise correlated strongly with the length measured by ultrasound ( $r=0.764$, $P=0.001$ ) (Figure 2O). Histologically determined area also correlated strongly with ultrasound-measured area $(\mathrm{r}=0.761, P=0.003)$ (Online Supplementary Figure S4D). Thus, histological evaluation of thrombus dimensions post-mortem was a reliable readout for the actual situation in vivo.

We then evaluated whether iron supplementation could reverse the increased thrombus size in ID (Experiment 2). Def animals that received placebo (Def) were anemic, with higher platelet counts as compared to control rats fed a normal diet and given the placebo (Con). Administration of $20 \mathrm{mg} / \mathrm{kg} \mathrm{BW}$ ferric carboxymaltose to iron-deficient animals $(\mathrm{Def}+\mathrm{Fe})$ reversed anemia and normalized platelet 
A

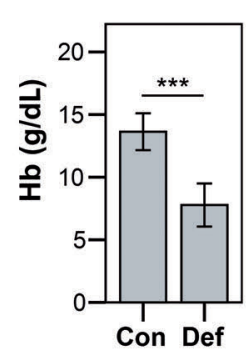

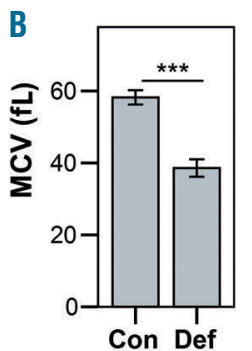
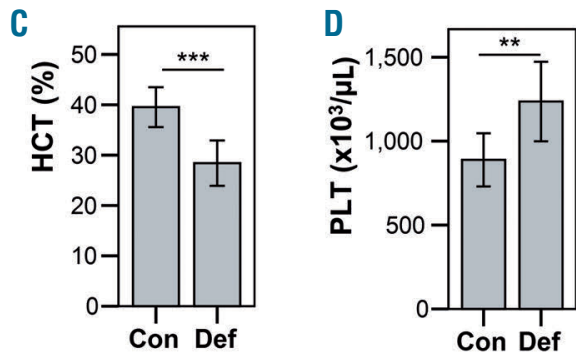

E
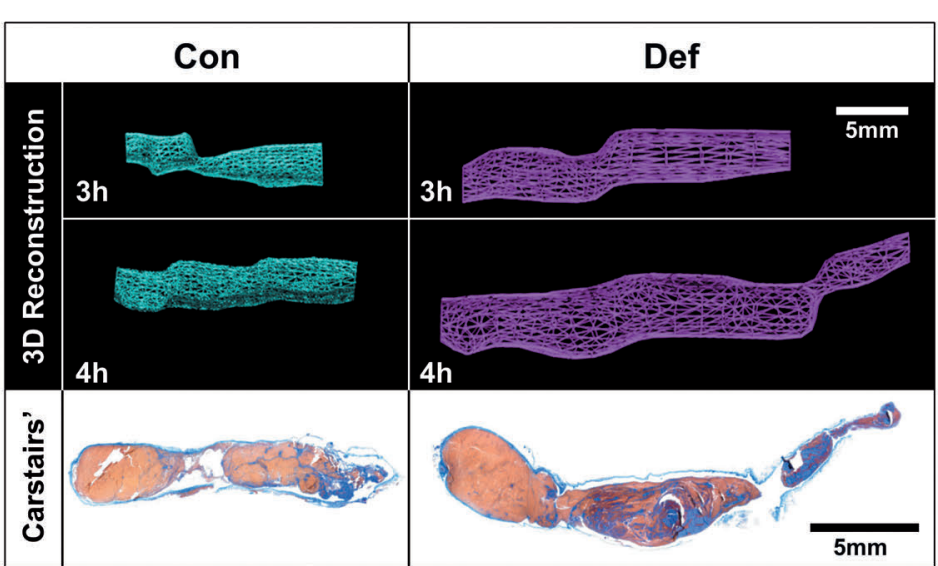

2 Bigation

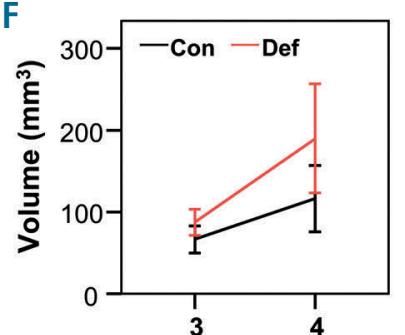

Time (h) post-ligation
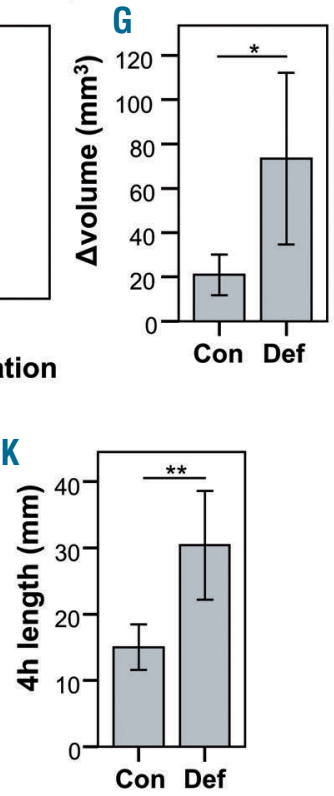

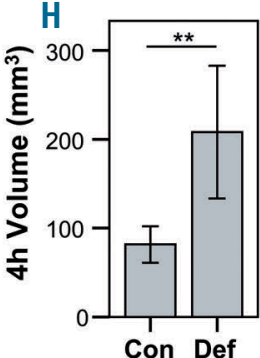

L

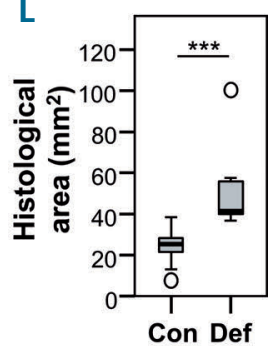

I
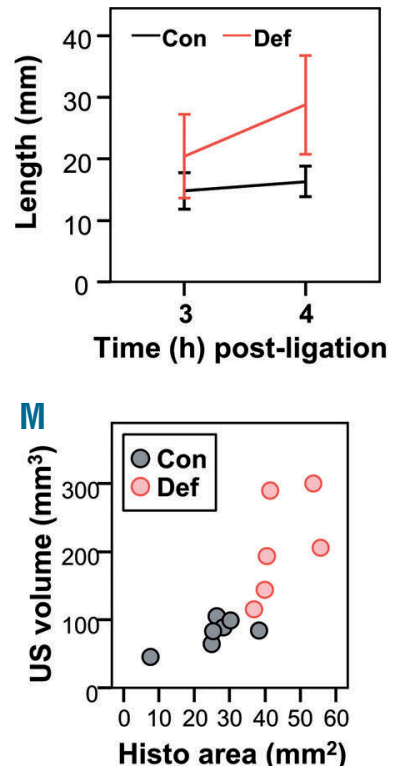
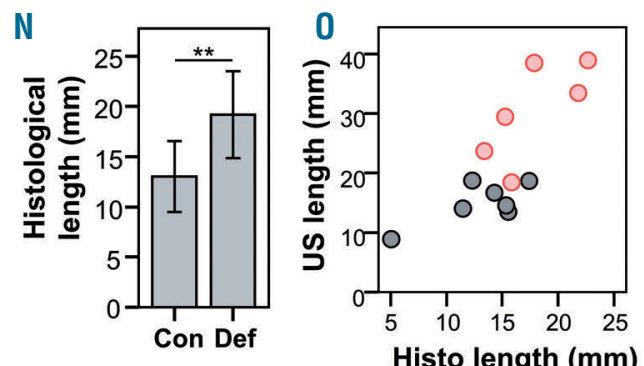

Figure 2. Iron deficiency augments venous thrombotic tendency. In experiment 1 , venous thrombosis was induced by stenosis of the inferior vena cava after animals had been on an iron-deficient diet (Def) for 7 weeks. A control group (Con) was fed a normal diet. (A-D) Hematologic parameters measured prior to surgery ( $n=7$ per group); hemoglobin (A), mean corpuscular volume (B), hematocrit $(C)$ and platelet count (D). (E) Representative three-dimensional images reconstructed from high frequency ultrasound scans at 3 and $4 \mathrm{~h}$ after ligation; and corresponding Carstairs staining of thrombi collected post-mortem. (F, G) Thrombus volume at 3 and $4 \mathrm{~h}$ after ligation ( $F$ ) and the change in volume over time $(G)$ ( $n=5$ per group, error bars: mean \pm standard deviation $[S D])$. (H) Final thrombus volume at $4 \mathrm{~h}$ ( $\mathrm{n}=6-7$ per group). (I, J) Thrombus length at 3 and $4 \mathrm{~h}$ after ligation (I) and the change in length over time $(J)(n=5$ per group, error bars: mean $\pm S D)$. (K) Final thrombus length at $4 h(n=6-$

7 per group). (L) Thrombus area measured on longitudinal histological sections ( $n=9$ per group). (M) Comparison of thrombus area measured histologically (Histo) and ultrasound (US) volume. (N) Thrombus length measured on longitudinal histological sections ( $n=9$ per group). (0) Comparison of thrombus length measured histologically and by US. $* P<0.05$, $* * P<0.01, * * * P<0.001$. Error bars: mean $\pm \mathrm{SD}$. $\mathrm{Hb}$ : hemoglobin, MCV: mean corpuscular volume, HCT: hematocrit, PLT: platelet count. 
counts (Figure 3B-E). Prussian blue staining of spleen and liver also showed increased iron staining with iron supplementation (red arrows, Online Supplementary Figure S5A). As in experiment 1, the average thrombus area, evaluated by histology, was larger in Def rats $\left(43.3 \pm 10 . \mathrm{mm}^{2}\right)$ than in Con rats $\left(24.9 \pm 12 . \mathrm{mm}^{2}, P=0.032\right)$. Iron replacement therapy reduced thrombus area to levels even lower than in Con animals (Def $+\mathrm{Fe}, 19.8 \pm 1.4 \mathrm{~mm}^{2}, P=0.011$ ) (Figure $3 \mathrm{G})$. Histologically determined thrombus length showed similar trends (Con: $11.2 \pm 4 \mathrm{~mm}$, Def: $16.3 \pm 2.3 \mathrm{~mm}$,
Def+Fe: $14.58 \pm 6 \mathrm{~mm})$, although the differences were not statistically significant (Online Supplementary Figure S5B).

Upon plotting thrombus area against platelet count in both experiments, it was apparent that the higher platelet counts in ID animals were related to larger resultant thrombus areas $(r=0.634, P=0.003$ ) (Figure $3 \mathrm{H})$. Def $+\mathrm{Fe}$ animals were more similar to control animals, reflecting the reduction in counts upon iron replacement therapy.

Carstairs method for fibrin and platelet staining ${ }^{22}$ showed thrombi composed primarily of erythrocytes
A

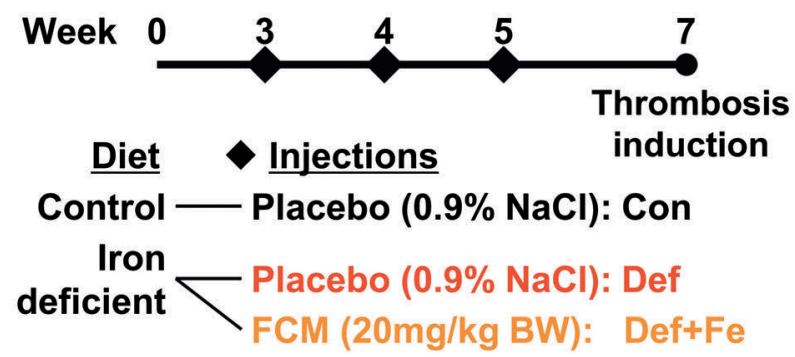

D
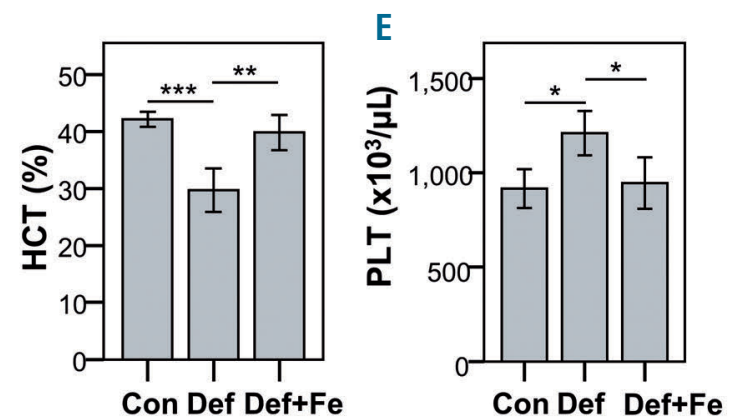

6

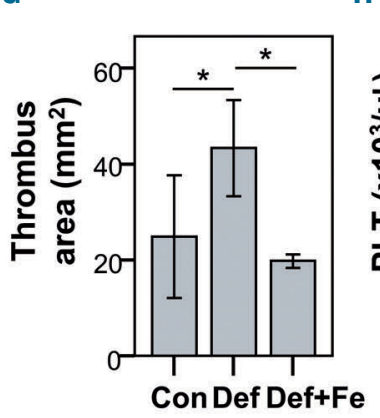

B

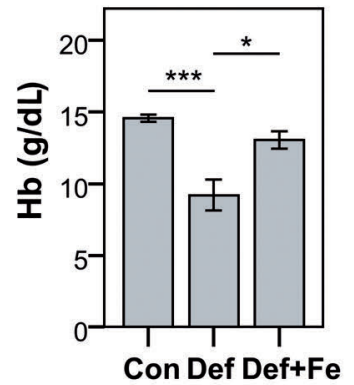

$\mathrm{F}$

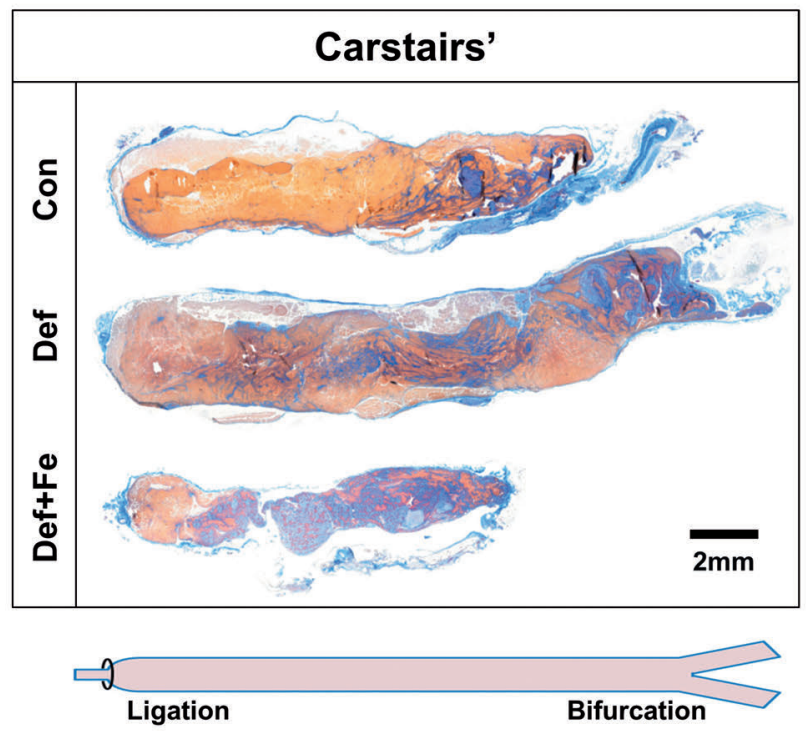

$\mathrm{H}$

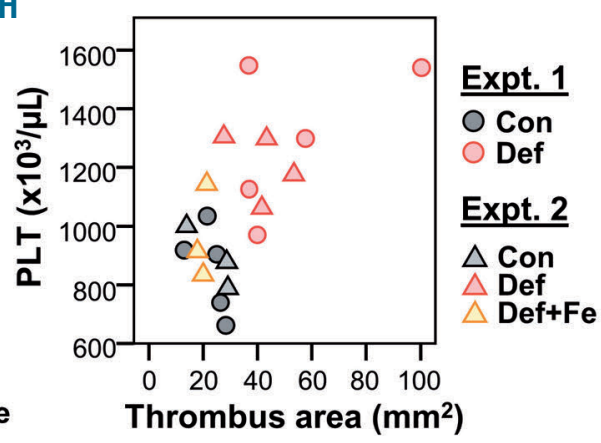

Figure 3. Iron replacement therapy reverses iron deficiency-induced increased venous thrombotic tendency. (A) Design of experiment 2. Rats were fed an iron-deficient diet (Def) for 7 weeks. and given three injections of ferric carboxymaltose (FCM) at the doses of 5, 10, or $20 \mathrm{mg} / \mathrm{kg}$ body weight (BW, +Fe5, +Fe10, +Fe20, respectively) or placebo $(0.9 \% \mathrm{NaCl})$. Animals fed a control diet and given injections of placebo formed the control group (Con). Venous thrombosis was induced after hematologic parameters had been measured. (B-E) Hematologic parameters measured prior to surgery ( $n=4$ per group): hemoglobin (B), mean corpuscular volume $(C)$, hematocrit (D) and platelet count (E). (F) Representative images of Carstairs-stained sections of thrombi. (G) Thrombus area measured on longitudinal sections ( $n=4-5$ per group). $(H)$ Thrombus area versus platelet counts of both experiments, when both measurements were available ( $n=3-5$ per group). $* P<0.05, * * P<0.01$, $* * * P<0.001$. Error bars: mean \pm standard deviation. Hb: hemoglobin; MCV: mean corpuscular volume; HCT: hematocrit, PLT: platelet count. 
(reddish-brown areas) with areas staining strongly for platelets (navy-blue areas) (Figures 2E, 3F, and 4A). The navy-blue areas on Carstairs staining correspond to areas staining positive for platelet receptor integrin alpha-IIb (ITGA2B) (Figure 4A and B). In both experiments, thrombi from Def animals had larger ITGA2B-positive areas (Figure 4B and C) as compared to those in the Con animals. Iron replacement therapy normalized these areas to control levels (Figure 4C). The larger ITGA2B areas also corresponded to higher platelet counts $(\mathrm{r}=0.537, P=0.015)$ (Figure 4D).

\section{Iron deficiency enhances thrombotic tendency in an} arterial model of thrombosis

In a separate experiment, the tendency to arterial thrombosis was evaluated using the ferric chloride model, in which time to thrombotic occlusion of the right common carotid artery after vascular damage was measured using a transonic flow probe. Thrombi were collected immediately after complete cessation of flow and evaluated histologically.

The model of IDA and thrombocytosis remained robust and replicable, and iron supplementation normalized hematologic parameters (Figure 5A, Online Supplementary Table S3). Similar to the results of the venous thrombosis model, thrombus area and length were larger in Def rats (area: $1.5 \pm 0.4 \mathrm{~mm}^{2}$; length: $3.7 \pm 1.0 \mathrm{~mm}$ ) than in Con rats (area: $0.9 \pm 0.3 \mathrm{~mm}^{2}, P=0.008$; length: $2.5 \pm 0.7 \mathrm{~mm}$, $P=0.034)$. Def + Fe thrombi tended to be smaller than Con thrombi, although the difference was not statistically significant (area: $1.1 \pm 0.4 \mathrm{~mm}^{2}, P=0.19$; length: $2.9 \pm 0.8 \mathrm{~mm}$, $P=0.27$ ) (Figure $5 \mathrm{~B}$ and $\mathrm{C}$ ).
A

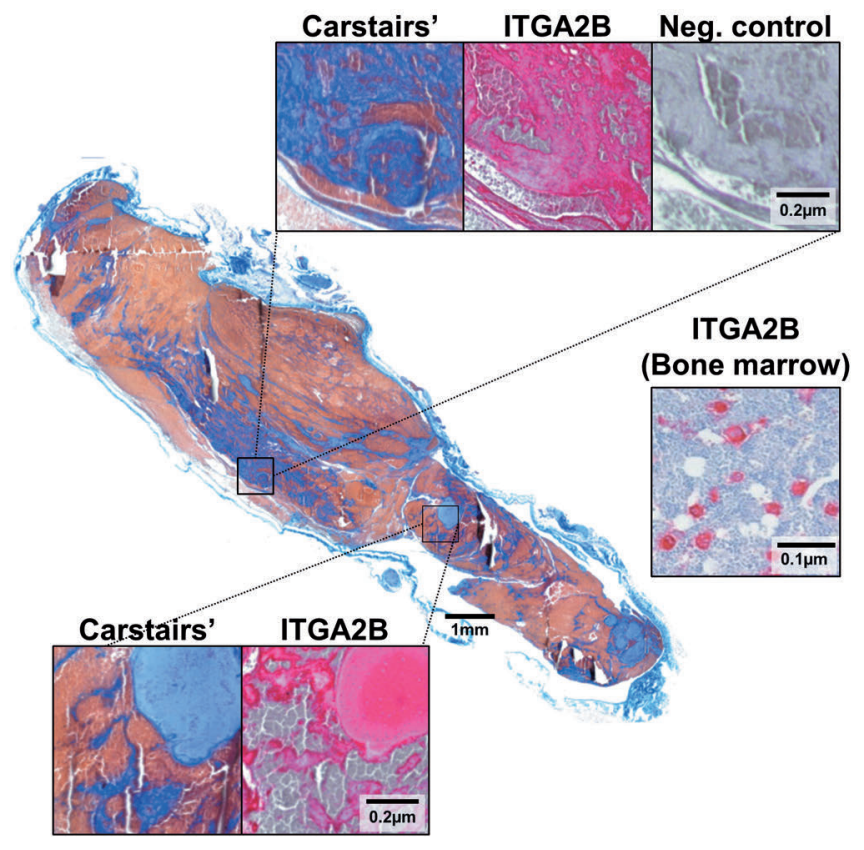

B

\section{Experiment 1 Experiment 2}
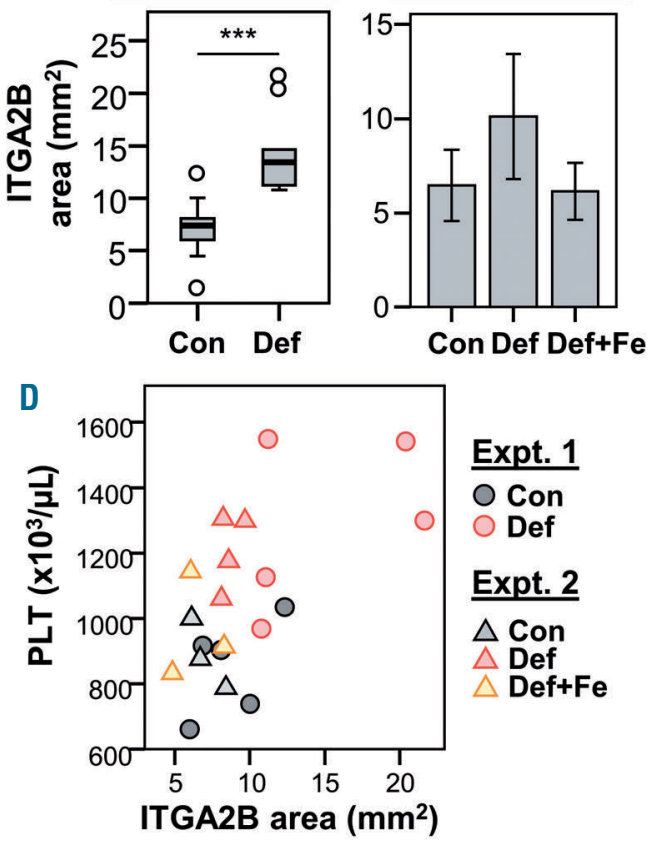

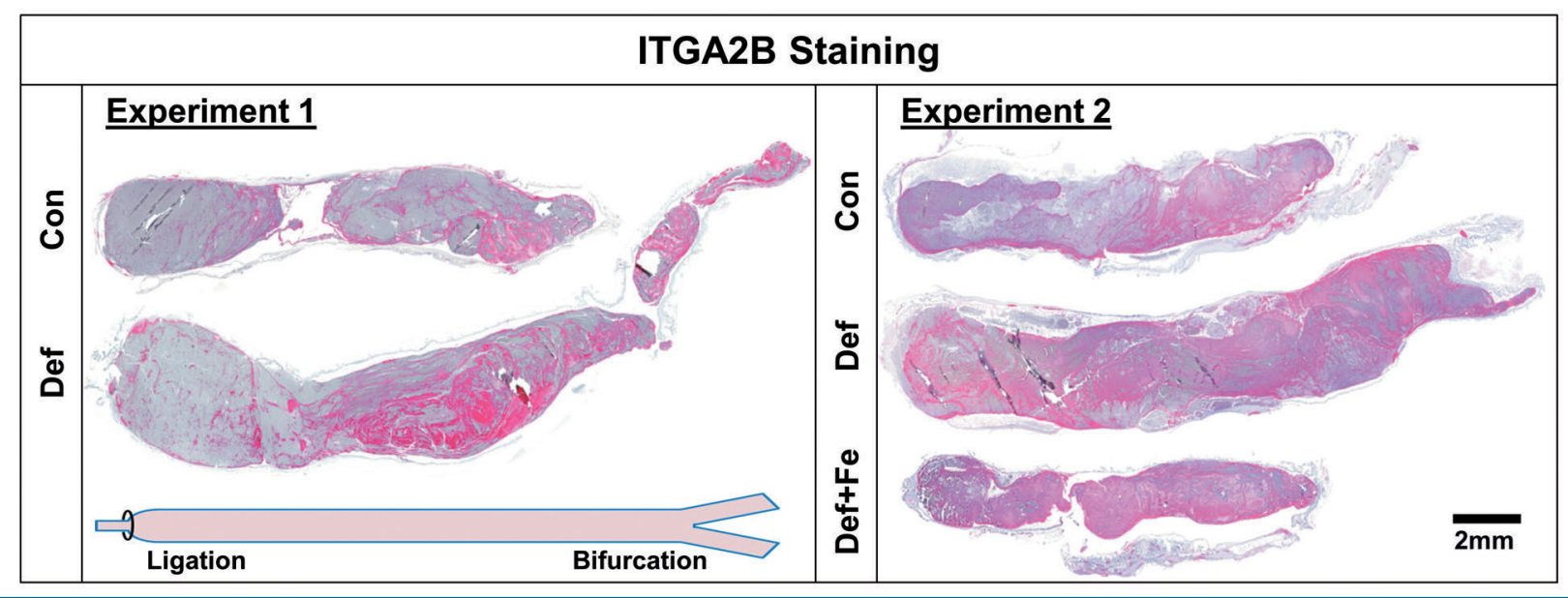

Figure 4. Platelet area of venous thrombi is larger in iron deficiency. (A) Carstairs staining correlated with ITGA2B immunohistochemical staining for venous thrombi. Bone marrow was used as a positive control for ITGA2B. (B) Representative images of ITGA2B staining of venous thrombi. (C) Area of thrombus staining positive for ITGA2B. Experiment 1: $n=9$, Experiment 2: $n=4-5$ per group. (D) ITGA2B-positive area versus platelet count of both experiments, when both measurements were available ( $n=3-5$ per group) $* * * P<0.001$. Error bars: mean \pm standard deviation. PLT: platelet count. Con: animals fed a normal diet and given placebo injections; Def: animals fed an iron-deficient diet; Def+Fe: animals fed an iron-deficient diet and then given injections of ferric carboxymaltose. 
A
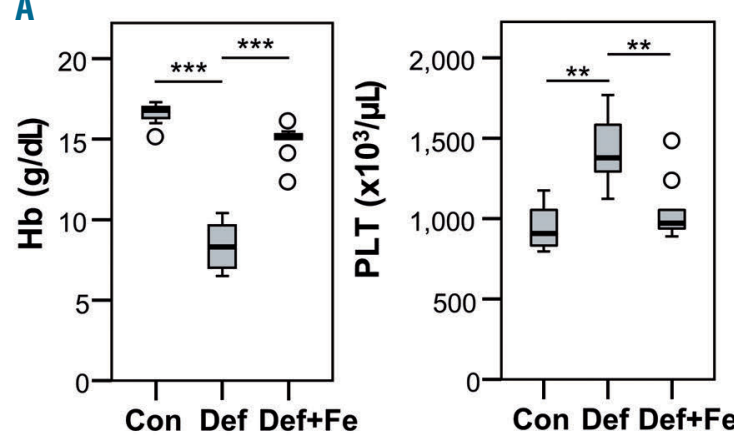

B

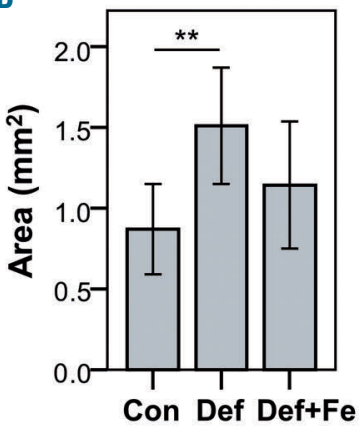

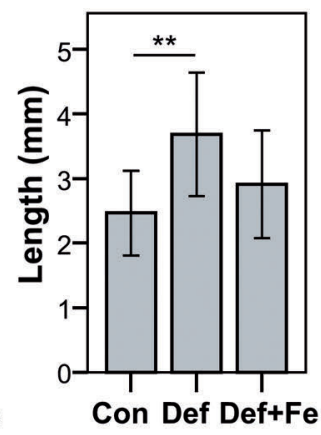

C

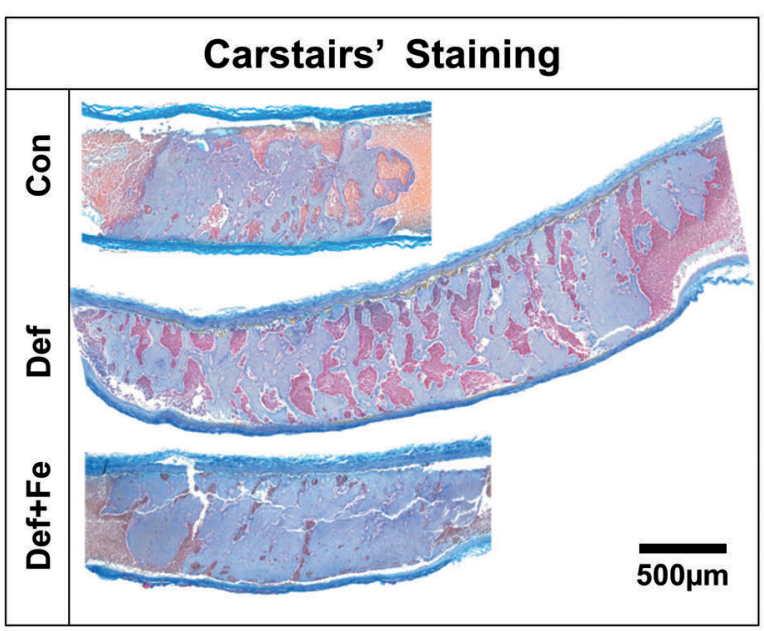

D

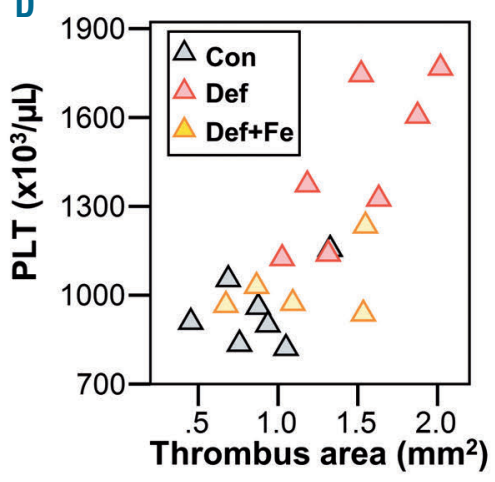

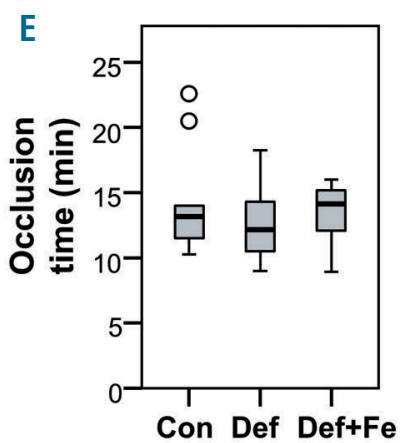
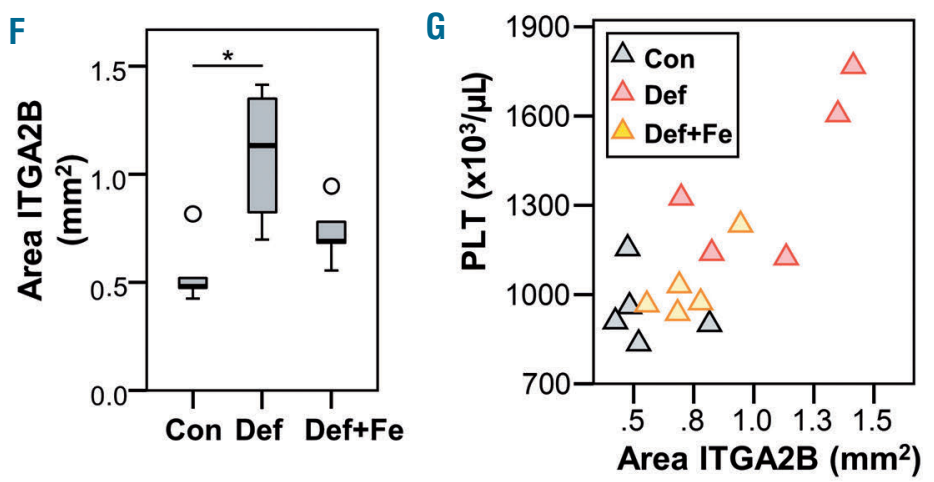

H

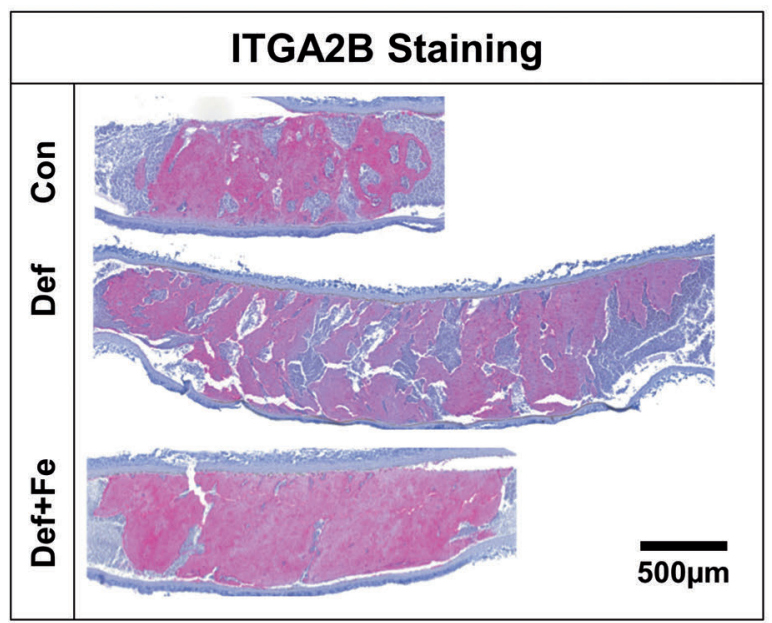

Figure 5. Iron deficiency enhances arterial thrombotic tendency. Arterial thrombosis was induced in animals fed an iron-deficient diet for 7 weeks and given three injections of ferric carboxymaltose at a dose of $20 \mathrm{mg} / \mathrm{kg}$ body weight or placebo $(0.9 \% \mathrm{NaCl})$. (A) Hemoglobin concentration and platelet counts prior to surgery ( $n=9-11$ per group). (B) Thrombus area and length measured on Carstairs-stained Iongitudinal sections of thrombi $(n=5-7$ per group). (C) Representative images of Carstairs-stained arterial thrombi. (D) Thrombus area versus platelet count $(n=5-7$ per group). (E) Time until cessation of carotid blood flow due to thrombus development ( $n=9-11$ per group). ( $F)$ Area of thrombus staining positive for ITGA2B ( $n=5$ per group). (G) ITGA2B-positive area versus platelet count ( $n=5$ per group). (H) Representative images of ITGA2B staining of arterial thrombi. $* P<0.05, * * P<0.01$, $* * * P<0.001$. Error bars: mean \pm standard deviation. $\mathrm{Hb}$ : hemoglobin; PLT: platelet count. Con: animals fed a normal diet and given placebo injections; Def: animals fed an iron-deficient diet; Def+Fe: animals fed an iron-deficient diet and then given injections of ferric carboxymaltose. 
Plotting thrombus area against platelet counts again showed that higher counts corresponded with a larger resultant thrombus area $(r=0.773, P<0.001)$ (Figure $5 \mathrm{D}$ ) and that values in Def $+\mathrm{Fe}$ animals were closer to Con values than those of Def animals. Interestingly, thrombus size was larger in Def rats despite the occlusion times being similar across the three groups (Figure 5E). This implies that the rate of thrombus growth was faster in Def animals.

In comparison to venous thrombi, arterial thrombi are composed predominantly of platelets (Figure $5 \mathrm{H}$, Online Supplementary Figure S6A). Accordingly, thrombi from Def rats had larger ITGA2B-positive areas as compared to those from Con or Def+Fe animals (Figure 5F). As in the venous thrombosis model, the larger ITGA2B areas corresponded to higher platelet counts $(\mathrm{r}=0.806, P<0.001)$ (Figure 5G).

In summary, in models of both venous and arterial thrombosis, ID was associated with thrombocytosis and increased thrombus size. Iron replacement therapy normalized these parameters to approach values in control animals, underscoring the iron dependency of the phenotype.

\section{Iron deficiency increases coagulability and platelet} activity, but impairs platelet function under shear flow

We further examined whether ID influences coagulability and platelet function in vitro, to gain insight into what could be contributing to the increased thrombus size in the thrombosis models. As in previous experiments, ID led to thrombocytosis, which resolved with iron replacement therapy (Figure 6A and B, Online Supplementary Table S3).

Upon thromboelastography, clot strength and the maximum rate of thrombus generation were higher in Def rats as compared to Con rats and normalized in response to iron $(\mathrm{Def}+\mathrm{Fe}$ ) (Figure 6C and D). The time to first formation of fibrin was shorter in Def animals and normalized on iron replacement therapy (Figure 6E). These parameters combined into an overall higher coagulation index in Def animals, as compared to Con or Def $+\mathrm{Fe}$ animals (Figure 6F).

We then evaluated platelet activity via flow cytometric measurement of P-selectin expression upon stimulation with $\mathrm{ADP}$ and thrombin. We found that expression of $\mathrm{P}-$ selectin was already higher in samples from Def animals, even without agonist stimulation (Baseline, Figure 7A). Upon stimulation with thrombin $(0.25 \mathrm{U} / \mathrm{mL})$, the expression of P-selectin was greater in Def than in Con or Def + Fe animals. In contrast, stimulation with ADP $(2.5$ $\mathrm{mM}$ ) increased P-selectin expression to a similar degree across all groups (Online Supplementary Figure S7A).

Plasma soluble P-selectin levels were higher in Def animals than in Con ones (Figure 7B), which corroborated the higher baseline platelet activity seen on flow cytometry. The levels normalized to Con levels in Def $+F e$ animals. In comparison, following arterial thrombosis, soluble Pselectin levels were lower in Def rats than in Con ones, whereas levels were similar across groups after venous thrombosis (Online Supplementary Figure S7B).

We then used the PFA-100 to measure platelet adhesion and aggregation under shear flow. Interestingly, $60 \%$ of the samples from Def animals did not occlude. Those that did occlude also took longer to do so than platelets from Con or Def + Fe animals (Figure 7C).

To evaluate whether there was a defect in platelet aggre- gation, whole blood samples were tested on a Multiplate Aggregometer with ADP and collagen as agonists. Aggregability (determined by the area under the curve) in response to $\mathrm{ADP}$ and collagen was similar across the groups, with a trend towards an increase in Def rats (Figure 7D). To assess whether there was a defect in adhesion, a static adhesion assay using fibrinogen as well as collagen-coated wells was performed. There were no observable differences in static adhesion between the groups for collagen or fibrinogen (Figure 7E). Stimulation with ADP did not result in any added effect (Online Supplementary Figure S7C). Platelet function was thus only impaired under shear flow, and otherwise appeared to be similar between the groups in assays without shear. We

A

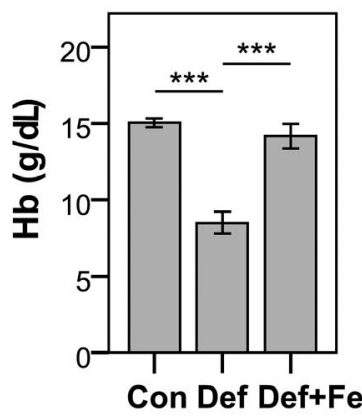

B
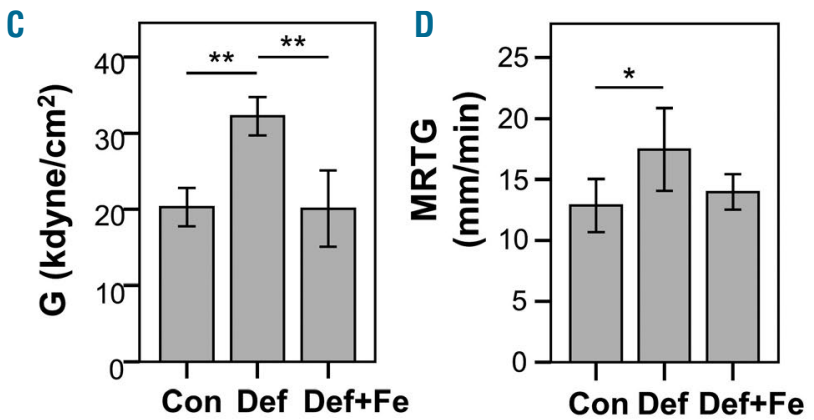

E
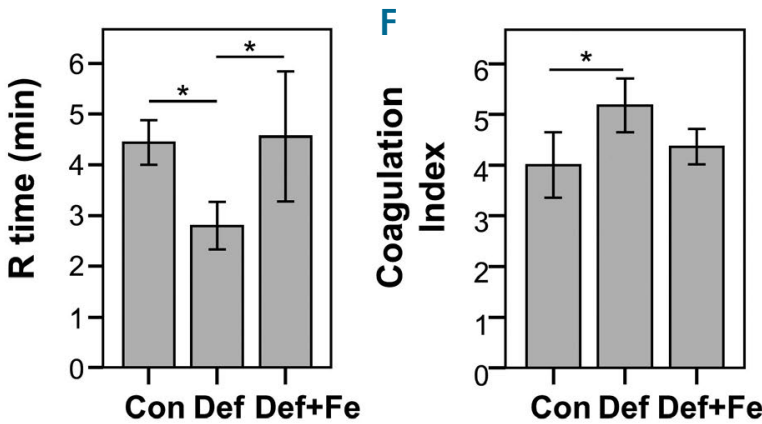

Figure 6. Iron deficiency augments clot dynamics. Animals were fed an irondeficient diet for 7 weeks and given three injections of ferric carboxymaltose at a dose of $20 \mathrm{mg} / \mathrm{kg}$ body weight or placebo $(0.9 \% \mathrm{NaCl})$. (A, B) Hemoglobin (A) and platelet counts $(B)$ at the end of the experiment ( $n=5$ per group). (C- $F$ ) Thromboelastography measurements of clot strength $(\mathrm{C})$, maximum rate of thrombus generation (D), time to clot initiation (E), and coagulation Index (F) ( $n=4-5$ per group). $P<0.05, * * P<0.01, * * * P<0.001$. Error bars: mean \pm standard deviation. Con: animals fed a normal diet and given placebo injections; Def: animals fed an iron-deficient diet; Def+Fe: animals fed an iron-deficient diet and then given injections of ferric carboxymaltose; Hb: hemoglobin; PLT: platelet count; G: clot strength; MRTG: maximum rate of thrombus generation; R: time to clot initiation. 
A

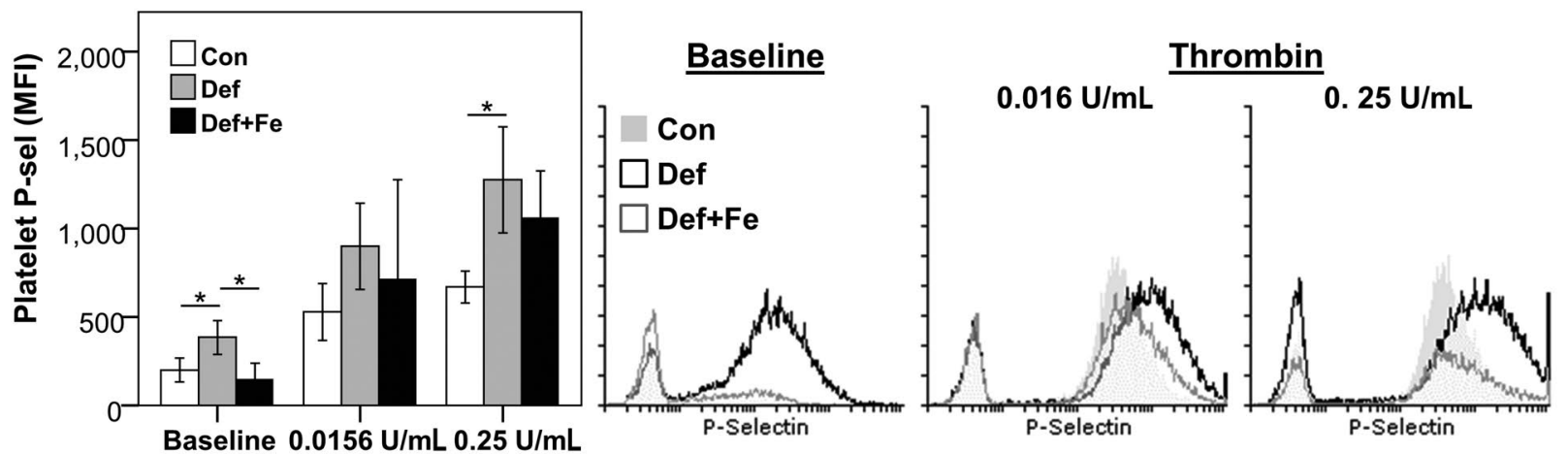

Thrombin

B

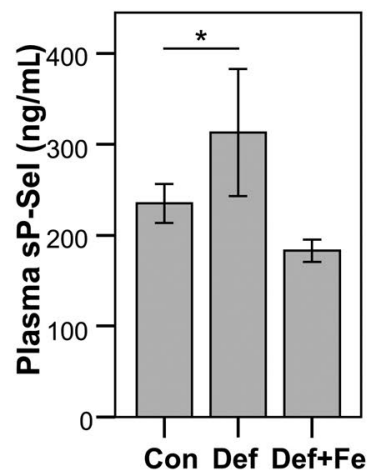

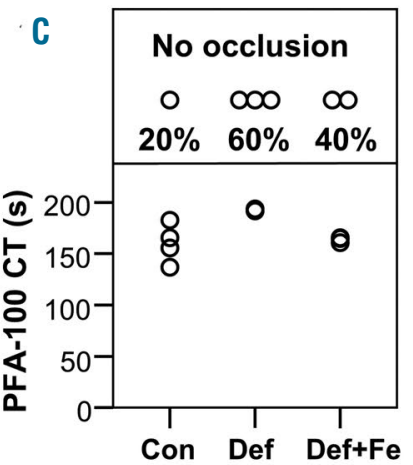
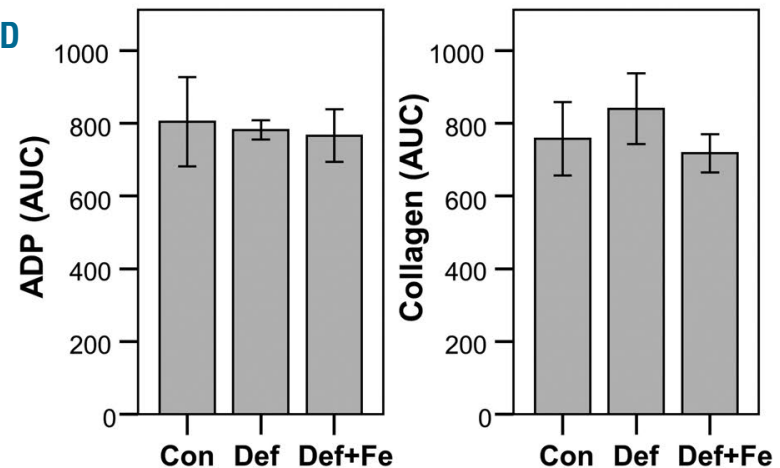

Static Platelet Adhesion

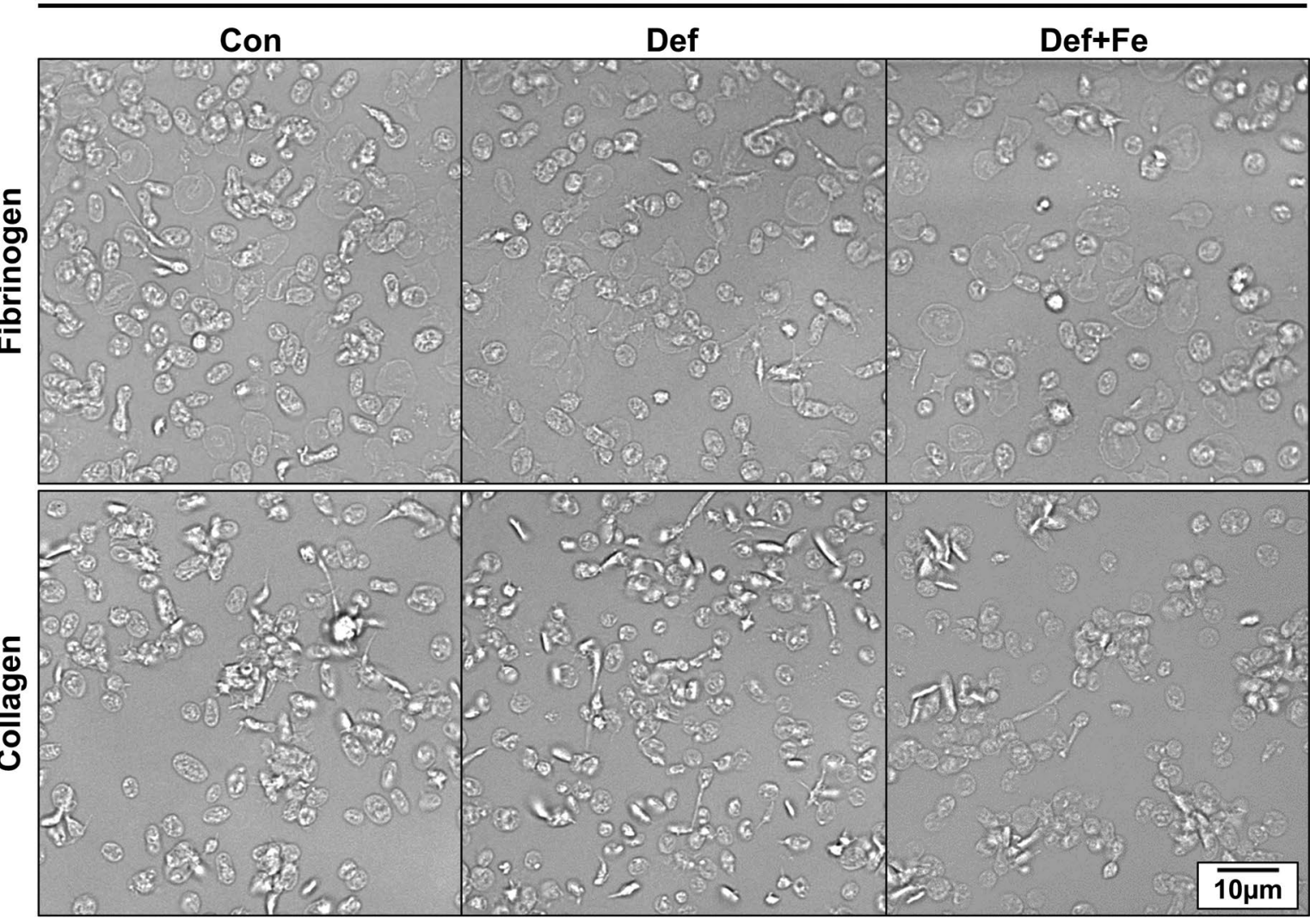

Figure 7. Iron deficiency increases the expression of P-selectin, without altering platelet aggregability or static adhesion. (A) Mean fluorescence intensity of P-selection expression on platelets before and after stimulation with thrombin $(0.0156 \mathrm{U} / \mathrm{mL}, 0.25 \mathrm{U} / \mathrm{mL})$. Error bars: mean \pm standard deviation $(\mathrm{SD})(\mathrm{n}=4$ per group). Representative histograms show the population shift. (B) Plasma P-selectin concentration determined by enzyme-linked immunosorbent assay ( $\mathrm{n}=4$ per group). (C) Platelet Function Analyzer closure time, a measurement of platelet adhesion under shear flow ( $\mathrm{n}=5$ per group), depicted as seconds until occlusion, or no occlusion. (D) Area under the curve measured from Multiplate whole blood impedance aggregometry after stimulation with ADP or collagen ( $n=4-5$ per group). ( $E$ ) Representative images of washed platelets adhering to a fibrinogen- or collagen-coated surface. $* P<0.05$. Error bars: mean \pm SD. P-sel: P-selectin; MFI: mean fluorescent intensity; sP-sel: soluble P-selectin; PFA-100 CT: Platelet Function Analyzer 100 closure time; AUC: area under the curve; Con: animals fed a normal diet and given placebo injections; Def: animals fed an iron-deficient diet; Def+Fe: animals fed an iron-deficient diet and then given injections of ferric carboxymaltose. 
theorized that there could be a defect in von Willebrand factor, and immunohistochemical staining of arterial thrombi did appear to be patchier in Def rats than in Con rats (Online Supplementary Figure S8A), which was not the case for venous thrombi (Online Supplementary Figure S8B).

In summary, baseline platelet activity was higher in ID, and platelet response to thrombin and clot dynamics were also augmented. Iron replacement therapy normalized these changes. Platelet aggregation to collagen and ADP and static platelet adhesion appeared to be similar across the groups. Platelet adhesion under shear flow appeared to be impaired, which could explain why occlusion times in the arterial model were similar across the groups. However, the net effect resulted in a greater thrombus size in both venous and arterial models.

\section{Discussion}

In this study we used animal models to investigate thrombotic tendency in ID. We found that IDA increases platelet numbers, blood coagulability, as well as baseline platelet activity. While these effects were protective, by augmenting hemostasis in response to tail injury, they also led to larger thrombi in both venous and arterial models of thrombosis. Alterations increasing the thrombotic tendency reversed upon iron replacement therapy, supporting the notion that IDA is a prothrombotic state.

We showed that platelet counts are consistently elevated in iron-deficient rats, confirming our previous findings, ${ }^{16}$ and further showed that these changes are reversible. Similar ID-induced thrombocytosis is common in humans and iron replacement therapy has been shown to reduce platelet counts in various groups of patients, including those with inflammatory bowel disease or chronic kidney disease, and even in iron-depleted blood donors. ${ }^{18,19,26-28}$

We have also shown that IDA in the absence of any other confounding illness can increase thrombus size in both arterial and venous animal models of thrombosis. While we are limited by current models in which thrombosis is induced rather than spontaneous, numerous clinical studies have found that ID may indeed be a risk factor for thrombotic events. ${ }^{7-13}$ In clinical studies, a large thrombus burden contributes to poor clinical outcome by increasing recurrence or mortality. ${ }^{29.31}$ As anemia and ID itself are known to be detrimental in cardiovascular disease, ${ }^{3}$ the alterations in platelet numbers and function could be other aspects of ID that contribute to adverse sequelae.

ID led to an increase in some parameters of the in vitro assays. Clot dynamics were augmented by ID, with an increase in clot formation as well as clot strength. Similar results were found in children, in whom IDA increased clot firmness on rotational thromboelastometry. ${ }^{32}$ Another study using a thrombin generation assay also found a decrease in coagulability in response to iron replacement therapy. ${ }^{33}$ This was likely due to an increase in the contribution of platelets to clot formation, which is also reflected by the increased platelet area seen on thrombus immunohistochemistry.

Baseline expression of P-selectin by platelets was also higher in ID, which suggests that circulating platelets are already partially activated. Stimulation with thrombin, but not ADP, increased P-selectin expression further. Baseline expression in control and iron-treated animals was similar, and the response to thrombin was diminished in comparison to that of animals with ID but still higher than that of controls. Our group previously showed that baseline and ADP-stimulated platelet P-selectin expression was higher in patients with inflammatory bowel disease who had ID than in those after iron replacement therapy. ${ }^{19}$ In another study on children with IDA, P-selectin was likewise elevated on agonist stimulation. ${ }^{34}$ Apart from platelet P-selectin, we also found in the current study that plasma levels of soluble P-selectin were increased in ID and diminished upon iron replacement therapy. Increasing soluble P-selectin by genetic engineering in mice leaves the animals in a procoagulant-prothrombotic state. . $^{3,36}$ This is relevant, as thrombocytosis and soluble P-selectin are predictive of VTE in cancer patients, ${ }^{37}$ who may also suffer from functional ID and anemia of chronic disease.

We did not find a significant difference in platelet aggregability to $\mathrm{ADP}$ or collagen in ID, although our previous study showed increased aggregability to ADP in platelets from rats after 2 weeks on an iron-deficient diet; this aggregability then diminished by 3 weeks. ${ }^{16}$ Several studies of patients have actually found impaired aggregability to ADP or collagen in IDA, ${ }^{34}$ which improved with iron replacement therapy. ${ }^{38-40}$ This occurred despite an increased platelet count in ID in some studies. ${ }^{38,39} \mathrm{We}$ found only one study that recorded an increase in aggregability. ${ }^{41}$ This could be due to methodological differences, as platelets resuspended in plasma were used in the previous animal study, ${ }^{16}$ whereas we used whole blood in order to mimic the intravascular environment better. However, it could also be due to differences in timing: perhaps aggregability increases in early ID, and diminishes over time.

Remarkably, we found impaired adhesion of platelets from iron-deficient animals under flow (as determed using the PFA-100), with no impairment in static adhesion tests. While hematocrit influences PFA-100 results, none of the samples tested exceeded the $25 \%$ cutoff, ${ }^{42}$ suggesting that the results may be due to a functional defect of the platelets. Similar results have been found in children with IDA, whose PFA-100 closure times were significantly longer than those of controls. ${ }^{34,43}$ This defect in adhesion under flow could explain why there were no differences in occlusion times, as the model of arterial thrombosis is subject to high shear flow. Nevertheless, both hemostasis and arterial thrombus size were augmented in ID. Perhaps the defect is compensated for by the increased platelet count.

We hypothesize that apart from increased platelet counts, there is also onging platelet activation in ID, resulting in an overall increased thrombotic tendency. Results of standard in vitro tests may be incongruous, but those of the thrombosis models are quite distinct. In one study, the risk of VTE in cancer patients was associated with a reduction in platelet responsiveness to PAR-1 and GPVI agonists. ${ }^{44}$ Further studies are needed to better define how ID influences platelet function, and what tests may be appropriate in this context. It is also unclear how ID augments platelet function, and whether this is a general feature of reactive thrombocytosis, or due to something specific to ID.

In conclusion, our study is the first to show that ID alone is sufficient to increase thrombotic tendency. ID consistently increases platelet counts, modulates platelet function, and increases thrombus size in both arterial and venous models of thrombosis. Iron replacement therapy reverses these changes, making it a viable strategy for the 
prevention of ID-related thrombotic disease. Patient blood management may thus be of particular importance in inflammatory bowel disease, cancer, chronic kidney disease, or even in the postoperative setting in patients with preexisting IDA or major surgical blood loss. ${ }^{45}$ Both IDA and anemia of chronic disease should be considered conditions in which there is a high risk of thrombosis. In chronic disease the induction of hepcidin blocks the release of iron from enterocytes, macrophages, and hepatocytes. Iron remains sequestered from the circulation, resulting in a functional ID. Clearly more studies are needed to explore the interplay between disease, ID, and thrombosis and to determine whether our results can be translated into the clinical setting.

\section{Disclosures}

$C G$ has received research support, lecturing or consulting honoraria from AOP Orphan Pharmaceuticals, Biogena Naturstoffe GmbH, Insightra Inc., Shire Inc., and Vifor Int.

\section{Contributions}

KJ designed and carried out experiments, performed the statistical analysis and wrote the manuscript. FL and AL contributed to the experimental design, performed animal experiments and critically reviewed the manuscript. GS performed the PFA-100 and Multiplate aggregometry measurements and critically reviewed the manuscript. PS contributed to establishing the arterial thrombosis model and flow measurements and critically reviewed the manuscript. A-MK contributed to establishing the venous thrombosis model and critically reviewed the manuscript. $A K$ assisted in experiments and critically reviewed the experiments. JF and TH contributed to venous thrombosis imaging and critically reviewed the manuscript. RE conceptualized and designed experiments, evaluated the statistical analysis, and critically reviewed the manuscript. VK contributed to the experimental design and editing the manuscript as well as critically reviewing it. CG supervised the study design, received funding, and edited and critically reviewed the manuscript.

\section{Acknowledgments}

We thank the Center for Anatomy and Cell Biology at the Medical University of Vienna for the use of their Slide Scanner. We also thank Irene Lang's group at the Division of Cardiology, Department of Internal Medicine II at the Medical University of Vienna for their initial guidance regarding the high frequency ultrasound analysis of venous thrombi.

\section{Funding}

This project was funded by the Austrian Science Fund (P 27681)

\section{References}

1. Camaschella C. Iron deficiency. Blood. 2019;133(1):30-39.

2. Evstatiev R, Gasche C. Iron sensing and signalling. Gut. 2012;61(6):933-952.

3. von Haehling S, Jankowska EA, van Veldhuisen DJ, Ponikowski P, Anker SD. Iron deficiency and cardiovascular disease. Nat Rev Cardiol. 2015;12(11):659-669.

4. Dan K. Thrombocytosis in iron deficiency anemia. Intern Med. 2005;44(10):1025-1026.

5. Keung YK, Owen J. Iron deficiency and thrombosis: literature review. Clin Appl Thromb Hemost. 2004;10(4):387-391

6. Munot P, De Vile C, Hemingway C, Gunny $\mathrm{R}$, Ganesan V. Severe iron deficiency anaemia and ischaemic stroke in children. Arch Dis Child. 2011;96(3):276-279.

7. Evstatiev R. [Iron deficiency, thrombocytosis and thromboembolism]. Wien Med Wochenschr. 2016;166(13-14):437-446.

8. Azab SF, Abdelsalam SM, Saleh SH, et al. Iron deficiency anemia as a risk factor for cerebrovascular events in early childhood: a case-control study. Ann Hematol. 2014;93(4):571-576.

9. Maguire JL, deVeber G, Parkin PC. Association between iron-deficiency anemia and stroke in young children. Pediatrics. 2007;120(5):1053-1057.

10. Chang YL, Hung SH, Ling W, Lin HC, Li HC, Chung SD. Association between ischemic stroke and iron-deficiency anemia: a population-based study. PLoS One. 2013;8(12): e82952.

11. Hung SH, Lin HC, Chung SD. Association between venous thromboembolism and iron-deficiency anemia: a population-based study. Blood Coagul Fibrinolysis. 2015;26(4):368-372.

12. Potaczek DP, Jankowska EA, Wypasek E, Undas A. Iron deficiency: a novel risk factor of recurrence in patients after unprovoked venous thromboembolism. Pol Arch Med Wewn. 2016;126(3):159-165
13. Song AB, Kuter DJ, Al-Samkari H Characterization of the rate, predictors, and thrombotic complications of thrombocytosis in iron deficiency anemia. Am J Hematol. 2020 Jul 3. doi: 10.1002/ajh.25925. Epub ahead of print. PMID: 32619079 .

14. Simanek R, Vormittag R, Ay C, et al. High platelet count associated with venous thromboembolism in cancer patients: results from the Vienna Cancer and Thrombosis Study (CATS). J Thromb Haemost. 2010;8(1):114-120.

15. Henry DH, Dahl NV, Auerbach MA Thrombocytosis and venous thromboembolism in cancer patients with chemotherapy induced anemia may be related to ESA induced iron restricted erythropoiesis and reversed by administration of IV iron. Am J Hematol. 2012;87(3):308-310

16. Evstatiev R, Bukaty A, Jimenez K, et al. Iron deficiency alters megakaryopoiesis and platelet phenotype independent of thrombopoietin. Am J Hematol. 2014;89(5):524529.

17. Jimenez K, Khare V, Evstatiev R, et al. Increased expression of HIF2alpha during iron deficiency-associated megakaryocytic differentiation. J Thromb Haemost 2015;13 (6):1113-1127.

18. Kulnigg-Dabsch S, Evstatiev R, Dejaco C, Gasche C. Effect of iron therapy on platelet counts in patients with inflammatory bowel disease-associated anemia. PLoS One. 2012;7(4):e34520.

19. Kulnigg-Dabsch S, Schmid W, Howaldt S, et al. Iron deficiency generates secondary thrombocytosis and platelet activation in IBD: the randomized, controlled thromboVIT trial. Inflamm Bowel Dis. 2013;19(8):1609-1616.

20. Diaz JA, Farris DM, Wrobleski SK, Myers $\mathrm{DD}$, Wakefield TW. Inferior vena cava branch variations in C57BL/6 mice have an impact on thrombus size in an IVC ligation stasis) model. I Thromb Haemost. 2015;13(4):660-664

21. Bonnard T, Hagemeyer CE. Ferric chloride- induced thrombosis mouse model on carotid artery and mesentery vessel. J Vis Exp. 2015;(100):e52838.

22. Carstairs KC. The identification of platelets and platelet antigens in histological sections. J Pathol Bacteriol. 1965;90(1):225-231.

23. Ruifrok AC, Johnston DA. Quantification of histochemical staining by color deconvolution. Anal Quant Cytol Histol. 2001;23(4): 291-299.

24. Schindelin J, Arganda-Carreras I, Frise E, et al. Fiji: an open-source platform for biological-image analysis. Nat Methods 2012;9(7):676-682.

25. Goodall AH, Appleby J. Flow-cytometric analysis of platelet-membrane glycoprotein expression and platelet activation. Methods Mol Biol. 2004·272·225-253

26. Hazara AM, Bhandari S. Intravenous iron administration is associated with reduced platelet counts in patients with chronic kidney disease. J Clin Phar Ther. 2015;40(1):20 23.

27. Yessayan L, Yee J, Zasuwa G, Frinak S Besarab A. Iron repletion is associated with reduction in platelet counts in non-dialysis chronic kidney disease patients independent of erythropoiesis-stimulating agent use: retrospective cohort study. BMC Nephrol. 2014:15:119.

28. Eder AF, Yau YY, West K. The effect of iron balance on platelet counts in blood donors. Transfusion. 2017:57(2):304-312.

29. Barreto AD, Albright $\mathrm{KC}$, Hallevi $\mathrm{H}$, et al Thrombus burden is associated with clinical outcome after intra-arterial therapy for acute ischemic stroke. Stroke. 2008;39(12):3231 3235.

30. Kalayci A, Gibson CM, Chi G, et al. Asymptomatic deep vein thrombosis is associated with an increased risk of death insights from the APEX trial. Thromb Haemost. 2018;118(12):2046-2052.

31. Sianos G, Papafaklis MI, Daemen J, et al. Angiographic stent thrombosis after routine use of drug-eluting stents in ST-segment elevation myocardial infarction: the impor- 
tance of thrombus burden. I Am Coll Cardiol. 2007;50(7):573-583.

32. Ozdemir ZC, Duzenli Kar Y, Gunduz E, Turhan AB, Bor O. Evaluation of hypercoagulability with rotational thromboelastometry in children with iron deficiency anemia. Hematology. 2018;23(9):664-668.

33. Nashashibi J, Avraham GR, Schwartz N, Awni Y, Elias M. Intravenous iron treatment reduces coagulability in patients with iron deficiency anaemia: a longitudinal study. Br J Haematol. 2019;185(1):93-101.

34. Yildirim ZK, Orhan MF, Buyukavci M. Platelet function alterations and their relation to P-selectin (CD62P) expression in children with iron deficiency anemia. Blood Coagul Fibrinolysis. 2011;22(2):98-101.

35. Kisucka J, Chauhan AK, Zhao BO, et al. Elevated levels of soluble P-selectin in mice alter blood-brain barrier function, exacerbate stroke, and promote atherosclerosis. Blood. 2009;113(23):6015-6022.

36. Andre P, Hartwell D, Hrachovinova I, Saffaripour S, Wagner DD. Pro-coagulant state resulting from high levels of soluble P-selectin in blood. Proc Natl Acad Sci U S A. 2000;97(25):13835-13840.

37. Ay C, Simanek R, Vormittag R, et al. High plasma levels of soluble P-selectin are predictive of venous thromboembolism in cancer patients: results from the Vienna Cancer and Thrombosis Study (CATS). Blood. 2008;112(7):2703-2708.

38. Kurekci AE, Atay AA, Sarici SU, Zeybek C Koseoglu V, Ozcan O. Effect of iron therapy on the whole blood platelet aggregation in infants with iron deficiency anemia. Thromb Res. 2000;97(5):281-285.

39. Caliskan U, Oner AF, Kabakus N, Koc H Diminished platelet aggregation in patients with iron deficiency anemia. Clin Appl Thromb Hemost. 1999;5(3):161-163.

40. Kabakus N, Yilmaz B, Caliskan U. Investigation of platelet aggregation by impedance and optic methods in children with iron deficiency anaemia. Haematologia. 2000;30(2):107-115.

41. Tekin D, Yavuzer S, Tekin M, Akar N, Cin
S. Possible effects of antioxidant status on increased platelet aggregation in childhood iron-deficiency anemia. Pediatr Int 2001;43 (1):74-77.

42. Harrison P, Lordkipanidzé M. Clinical Tests of Platelet Function: Academic Press, 2013

43. Mokhtar GM, Ibrahim WE, Kassim NA, Ragab IA, Saad AA, Abdel Raheem HG Alterations of platelet functions in children and adolescents with iron-deficiency anemia and response to therapy. Platelets. 2015;26(5):448-452.

44. Riedl J, Kaider A, Marosi C, et al. Decreased platelet reactivity in patients with cancer is associated with high risk of venous thromboembolism and poor prognosis. Thromb Haemost. 2017;117(1):9098.

45. Musallam KM, Tamim HM, Richards T, et al. Preoperative anaemia and postoperative outcomes in non-cardiac surgery: a retrospective cohort study. Lancet. 2011;378 (9800):1396-1407. 